\title{
Studies on the ns-IR-Laser-Induced Plasma Parameters in the Vanadium Oxide
}

\author{
Arnab Sarkar, Raju V. Shah, D. Alamelu, and Suresh K. Aggarwal \\ Fuel Chemistry Division, Bhabha Atomic Research Centre, Mumbai 400 085, India \\ Correspondence should be addressed to Arnab Sarkar, asarkar@ymail.com and Suresh K. Aggarwal, skaggr2002@gmail.com \\ Received 15 June 2011; Accepted 12 August 2011 \\ Academic Editor: Ali Hussain Reshak \\ Copyright () 2011 Arnab Sarkar et al. This is an open access article distributed under the Creative Commons Attribution License, \\ which permits unrestricted use, distribution, and reproduction in any medium, provided the original work is properly cited.
}

\begin{abstract}
We report spectroscopic studies of laser-induced plasma (LIP) produced by ns-IR-Nd:YAG laser light pulses of different energies onto four different oxides of vanadium $\left(\mathrm{VO}, \mathrm{V}_{2} \mathrm{O}_{3}, \mathrm{VO}_{2}\right.$, and $\left.\mathrm{V}_{2} \mathrm{O}_{5}\right)$ in air under atmospheric pressure. For each oxide with a different oxidation state of vanadium, both electron density and plasma temperature were calculated for different time delays and laser pulse energies. The plasma temperature was determined from Boltzmann plot method, whereas the electron number density was estimated from the Saha equation. The decay rates for plasma temperature as well as electron density were observed to follow power law and were independent of the nature of vanadium oxide. These investigations provide an insight to optimize various parameters during LIBS analysis of vanadium-based matrices.
\end{abstract}

\section{Introduction}

Laser-induced breakdown spectroscopy (LIBS) is an elemental analysis technique based on the excitation of atoms present on the sample surface, by focusing a pulsed laser beam. Material amounts ranging from ng to $\mu \mathrm{g}$ are ablated in this process producing a microplasma which can be characterized by several of parameters. After the creation of the plasma, electromagnetic radiation is emitted as a consequence of several processes, namely, bremsstrahlung, recombination and de-excitation of atoms and ions occurring inside the plume [1]. De-excitation of atoms and ions leads to emission of light of characteristic frequency which can be used for both qualitative and quantitative determinations. LIBS has several attractive features like the ability to analyze nonconducting and conducting material in any phase (solids, gases, and liquids) as well as refractory materials which are difficult to digest or dissolve (ceramics, superconductors, etc.). The rapidness of analysis and multielemental analyzing capability make LIBS a highly useful technique for trace elemental determination.

Several experimental parameters are reported to affect the LIBS analysis. These include the effect of laser wavelength [2], pulse energy [3], pulse duration and shape $[4,5]$, and the acquisition time delay. Cabalin and Laserna studied the effect of thermal property of material on threshold laser fluence variation [6]. The different phases of iron oxide were found to have different plasma characteristics [7]. To the best of our knowledge, the effect of different oxidation states of an element present in the sample on the plasma characteristics has not been reported.

Theoretically, the information regarding laser-induced plasma (LIP) can be obtained by solving a complex system of equations describing all the reactions, for example, ionization, dissociation, recombination, elastic and inelastic collisions, radiative emission, photon reabsorption, bremstrahlung that occur in the plasma, and the respective reactions' rates. The solution for such an equation is computationally tedious, and many times, all the relevant data needed for such computation cannot be obtained during experiments. Therefore, thermodynamical approach is usually preferred. The principle for characterization of LIP by this approach was described by Adrain and Watson [8] and is based on quantification of main physical parameters like the plasma temperature $\left(T_{e}\right)$, the electron density $\left(N_{e}\right)$, and the number densities of the ionic and the atomic species present in the plasma. These measurements allow improving the various applications of these parameters (namely, optimizing the experimental parameters) and are also useful for a better 
understanding of the complex ablation mechanism. Analyses of the spectral lines can give information about the physical state of the emitting species without, in any way, interfering with the plasma. The use of laser-based optical techniques in recent years has replaced spectroscopy as a diagnostic tool to some extent. Nevertheless, spectroscopy still plays a major role in studying the physical processes occurring in the plasma.

$N_{e}$ can be measured using optical emission plasma spectroscopy [9], Langmuir probe [10, 11], microwave and laser interferometry [12], and Thomson scattering [13]. Thomson scattering is the most direct method for such measurement, while the optical emission spectroscopy (using either Stark broadening of spectral lines or the Saha-Boltzmann equation) is the simplest as far as the instrumentation is concerned.

The $T_{e}$ is a key plasma parameter when local thermodynamic equilibrium (LTE) condition is satisfied by the plasma. $T_{e}$ determines the distribution of the species at different excitation levels satisfying the Boltzmann equation and the ionization equilibrium satisfying the Saha equation under the existence of the LTE. For the LTE to exist in the plasma, collisions with electrons have to dominate over the radiative processes, and this requires a sufficiently large electron density. For LTE to exist, a criterion was proposed by McWhirter [14] based on a critical electron density, which required the collision rate to be at least ten times of the radiative rate. For an energy gap difference of $\Delta \mathrm{E}$ $(\mathrm{eV})$ between the two neighboring states with an allowed transition and $T_{e}$ in Kelvin unit, the criterion for LTE is

$$
N_{e} \geq 1.6 \times 10^{18} T_{e}^{1 / 2}(\Delta E)^{3} m^{-3}
$$

The most widely used method for determination of plasma temperature in LTE plasma is based on the fact that the number densities in various excited states follow Boltzmann distribution, and hence, the plasma temperature of the line emissions can be measured from a single species. The Boltzmann plot method provides a single value of the plasma temperature of the plasma.

The aim of the present work was to study the LIP parameters of vanadium in four different vanadium oxides (VOs). The VOs plasma were generated by the fundamental (1064 nm) wavelength of a Q-switched Nd:YAG laser. The plasma temperature and the electron density were determined from the Boltzmann plot and the Saha-Boltzmann equation, respectively. The variation of LIP parameters with acquisition time delay was also estimated using the temporal analysis of the emission lines. In addition, the effect of laser energy on the LIP parameters of vanadium monooxide $(\mathrm{VO})$ and vanadium trioxide $\left(\mathrm{V}_{2} \mathrm{O}_{3}\right)$ was also studied. The studies reported in this work will help to optimize different experimental parameters, for example, laser energy, acquisition delay, and so forth, for LIBS analysis of high purity vanadium based materials like crystals, glasses, or nanocomposites, and so forth.

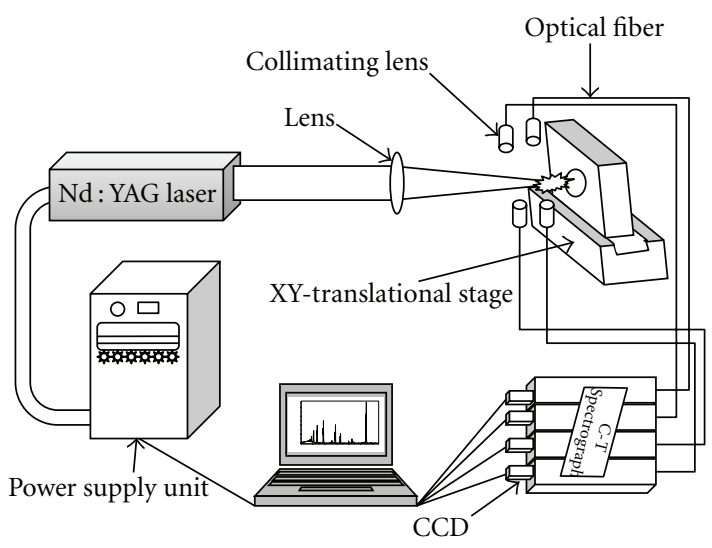

FIgURe 1: Schematic diagram of the LIBS setup.

\section{Experimental}

2.1. Instrumentation. Spectrolaser $1000 \mathrm{M}$, from Laser Analysis Technologies (now known as XRF scientific), Victoria, Australia, was used. A schematic diagram of the experimental setup was shown in Figure 1. The LIBS instrument was an integrated analysis system comprising an excitation laser, optical fibers, optical spectrograph, and charge-coupleddevice (CCD) array camera and is fully software controlled through a PC. The laser beam from a Q-switched Nd:YAG laser of $7 \mathrm{~ns}$ pulse width was capable of delivering maximum laser energy of $200 \mathrm{~mJ}$ at $1064 \mathrm{~nm}$ wavelength at a repetition rate of $10 \mathrm{~Hz}$. For the present study, laser pulses at a repetition rate of $1 \mathrm{~Hz}$ were used for the ablation of the target. The laser was focused on to the sample by a plano-convex lens of $5 \mathrm{~cm}$ focal length. The laser spot area is estimated to be $8 \times 10^{-9} \mathrm{~m}^{2}$. The sample was located on a fast XYtranslational stage, which moves the sample between two successive laser pulses, exposing a new and fresh region of the sample for each laser pulse. The emission is focused to an end of a fiber optic fiber (core diameter $200 \mu \mathrm{m}$ ) having a collimating lens $\left(0-45^{\circ}\right.$ field of view) placed at an angle of $45^{\circ}$ to the direction of the plasma expansion and was transmitted to the entrance slit of spectrographs in Czerny-Turner (C-T) configuration. Four separate optical cables connected to four spectrographs were present which are individually calibrated for specific wavelength range, giving a total spectral range of $180-850 \mathrm{~nm}$ at a spectral resolution of $0.6 \mathrm{~nm}$ at $300 \mathrm{~nm}$. Each spectrograph was equipped with 2048 element linear CCD array detector. The output from each of the four CCDs was digitized by an analog to digital convertor (ADC) circuit. The plasma light in the entire spectral range is thus recorded simultaneously for each laser pulse. The acquisition of the spectrum can be done at any fixed time delay between plasma ignition and recording of the emission spectrum. The average spectra resulting from the accumulation of 30 laser shots were used for analysis in the present work.

2.2. Sample Preparation and Analysis. Four vanadium oxide samples, namely, $\mathrm{VO}, \mathrm{V}_{2} \mathrm{O}_{3}, \mathrm{VO}_{2}$, and $\mathrm{V}_{2} \mathrm{O}_{5}(99.9 \%$ pure) were procured from Alfa Aeser, India. The oxide samples were homogeneously mixed with high purity of $99.5 \%$ boric 
TABLE 1: Composition of the vanadium oxide samples used.

\begin{tabular}{lccccr}
\hline \multirow{2}{*}{ Sample } & \multirow{2}{*}{ Oxide taken } & \multicolumn{2}{c}{ Amount taken $(\mathrm{gm})$} & Total amount $(\mathrm{gm})$ & \% of V \\
\hline 1 & $\mathrm{VO}$ & 0.6543 & 1.0065 & 1.661 & 49.79 \\
2 & $\mathrm{~V}_{2} \mathrm{O}_{3}$ & 0.7356 & 0.9995 & 1.735 & 50.00 \\
3 & $\mathrm{VO}_{2}$ & 0.8134 & 1.0037 & 1.817 & 49.96 \\
4 & $\mathrm{~V}_{2} \mathrm{O}_{5}$ & 0.8913 & 1.0027 & 1.894 & 49.93 \\
\hline
\end{tabular}

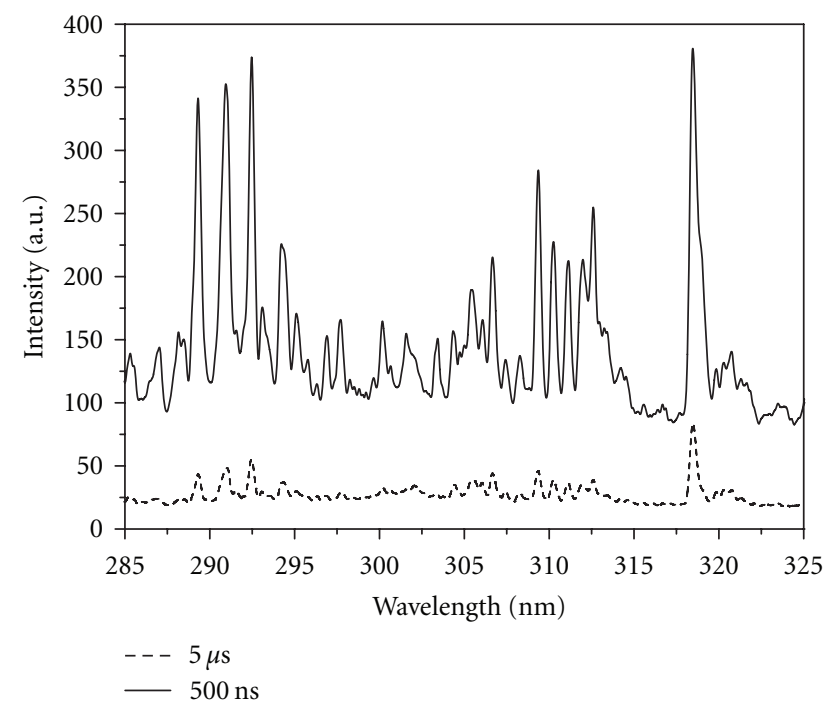

FIgURe 2: Typical LIBS spectra of VO at a laser energy $70 \mathrm{~mJ}$ and acquisition time delay of $500 \mathrm{~ns}$ (solid), $5 \mu \mathrm{s}$ (dash).

acid powder (S.D. Fine-Chem. Ltd., India) used as a binder, for $15 \mathrm{~min}$ by blending and grinding thoroughly to obtain homogeneous mixture. Mixed powder samples were then pelletized to $3 \mathrm{~cm}$ diameter pellets by applying a pressure of $2 \times 10^{9} \mathrm{~Pa}$ for $3 \mathrm{~min}$. The amount of oxide in each sample was adjusted in such a way that the amount of elemental vanadium remained constant $( \pm 0.15 \%)$ in each sample pellet. Table 1 gives the compositional data of the samples prepared and used in this work.

\section{Results and Discussion}

3.1. The Intensity Profile. Emission spectra of the different VOs were recorded at different acquisition delay times $(0-$ $8 \mu \mathrm{s})$ and different laser energies (50, 70, and $95 \mathrm{~mJ}$ ). Typical emission spectra at delay times of $500 \mathrm{~ns}$ and $5 \mu \mathrm{s}$ at a laser energy of $70 \mathrm{~mJ}$ are shown in Figure 2. It is seen that at the early stages of the plasma evolution; that is, at short acquisition delay times, the continuum emission is high, due to mechanisms involving free-free transition of electrons and radiative recombination, and therefore, the spectral information about atomic and ionic species will be inaccurate at short delay times.

Figure 3 shows the temporal emission behavior, that is, the normalized intensity monitored against acquisition delay observed for different vanadium oxides at $\mathrm{V}$ (I) $318.54 \mathrm{~nm}$

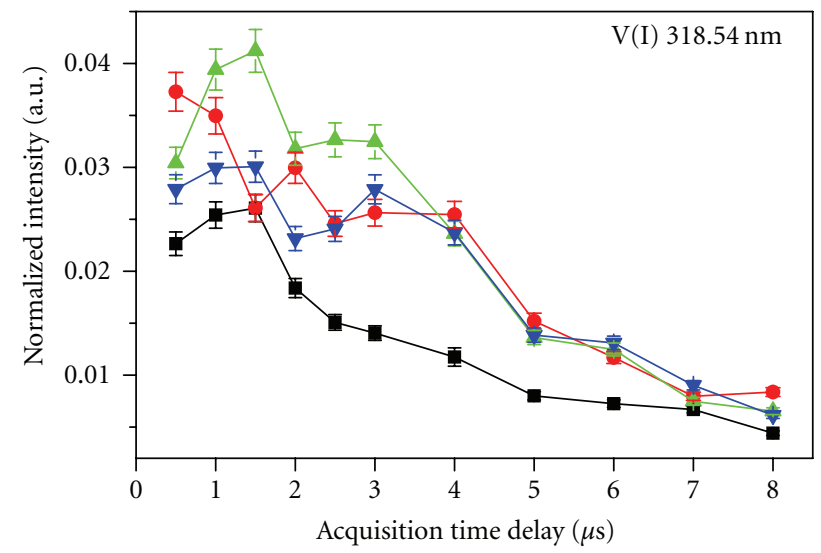

(a)

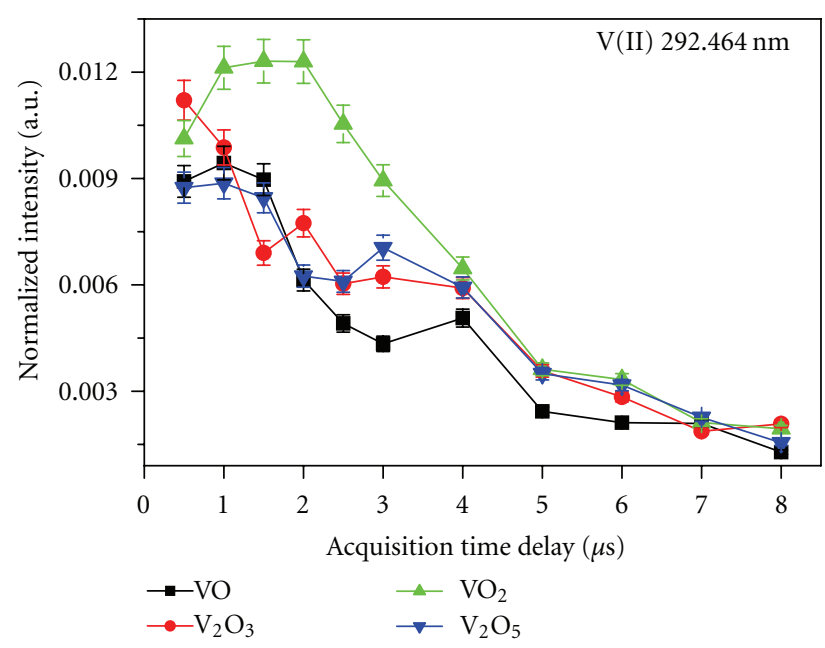

(b)

FIGURE 3: The temporal profile of $\mathrm{V}(\mathrm{I}) 318.54 \mathrm{~nm}$ and $\mathrm{V}(\mathrm{II})$ $292.464 \mathrm{~nm}$ LIP normalized emission intensity for different VOs at $70 \mathrm{~mJ}$. Normalization was done w.r.t. total integrated intensity of spectrograph.

and V(II) $292.464 \mathrm{~nm}$ at $70 \mathrm{~mJ}$. Normalization was done with respect to the total integrated intensity of the particular spectrograph used to monitor that particular emission line. For example, V(I) $318.54 \mathrm{~nm}$ and V(II) $292.464 \mathrm{~nm}$ emission lines were normalized with the total integrated intensity of the spectrograph-I, since this was used for monitoring of emission lines from 180-330 $\mathrm{nm}$. The existence of a number of humps is attributed to the possible different effects of the various physical processes governing the plasma behavior. It is seen that for $\mathrm{V}_{2} \mathrm{O}_{3}, \mathrm{VO}_{2}$, and $\mathrm{V}_{2} \mathrm{O}_{5}$, the atomic line 
TABLE 2: Chosen emission lines for $\mathrm{V}$ and their spectroscopic data [15].

\begin{tabular}{|c|c|c|c|c|c|c|}
\hline \multirow{2}{*}{ Species } & \multirow{2}{*}{ Wavelength (nm) } & \multirow{2}{*}{ Transition coefficient $\left(A_{i j}\right)\left(10^{8} \mathrm{~s}^{-1}\right)$} & \multicolumn{2}{|c|}{ Energy level } & \multirow[b]{2}{*}{$g_{j}$} & \multirow[b]{2}{*}{$g_{i}$} \\
\hline & & & Lower $\left(E_{j}\right)\left(\mathrm{cm}^{-1}\right)$ & $\operatorname{Upper}\left(E_{i}\right)\left(\mathrm{cm}^{-1}\right)$ & & \\
\hline $\mathrm{V}(\mathrm{II})$ & 292.464 & 1.2 & 2968.22 & 37150.51 & 9 & 9 \\
\hline $\mathrm{V}(\mathrm{I})$ & 305.633 & 1.3 & 0 & 32738.13 & 4 & 4 \\
\hline $\mathrm{V}(\mathrm{I})$ & 306.638 & 2.1 & 552.96 & 33155.30 & 10 & 10 \\
\hline $\mathrm{V}(\mathrm{I})$ & 318.540 & 2.7 & 552.96 & 31937.27 & 10 & 12 \\
\hline $\mathrm{V}(\mathrm{II})$ & 366.940 & 0.13 & 20363.23 & 47607.79 & 13 & 13 \\
\hline $\mathrm{V}(\mathrm{I})$ & 370.358 & 0.92 & 2424.78 & 29418.07 & 10 & 8 \\
\hline $\mathrm{V}(\mathrm{I})$ & 384.075 & 0.55 & 323.46 & 26352.65 & 8 & 6 \\
\hline $\mathrm{V}(\mathrm{II})$ & 395.197 & 0.11 & 11908.27 & 37204.98 & 5 & 7 \\
\hline $\mathrm{V}(\mathrm{I})$ & 440.764 & 0.44 & 2311.36 & 24992.88 & 8 & 8 \\
\hline
\end{tabular}

((V(I) $318.54 \mathrm{~nm})$ shows a sharp decrease of emission intensity after the gate delay of 4-5 $\mu$ s, in sharp contrast of $\sim 1.5 \mu$ s for VO. Ionic line shows more or less similar decay patterns of intensities from different oxides with faster decay than that of atomic line due to the recombination processes of electrons and ions. The analysis carried out at 50 and $95 \mathrm{~mJ}$ also exhibited the similar temporal profile except for differences in intensities magnitude which showed the order $95 \mathrm{~mJ}>70 \mathrm{~mJ}>50 \mathrm{~mJ}$. In case of ionic line, there was no difference in the line intensity at the different laser energies for delay times $>5 \mu$ s in view of their faster decay.

Nasrazadani and Namduri [7] reported that Fe present in a different matrix having similar composition but different phase will show different temporal decay pattern. The four VOs selected for this study also have different phases, that is, cubic for $\mathrm{VO}$, rhombohedral for $\mathrm{V}_{2} \mathrm{O}_{3}$, tetrahedral for $\mathrm{VO}_{2}$, and orthorhombic for $\mathrm{V}_{2} \mathrm{O}_{5}$. The different patterns observed in the emission lines in different VOs used in the present study also indicate the effect of phase difference among the samples. The ionic and atomic temporal decay profiles were also found to be different for different VOs. In addition, the photo-induced nonlinear optical effects [16], which are particularly sensitive to the oxides, could also be responsible for these differences.

3.2. Plasma Temperature. The $T_{e}$ was determined via the well-known Boltzmann plot method from the relative emission line intensities, provided that their transition probabilities $\left(A_{i j}\right)$ from a given excitation state are known. Under the LTE conditions in a plasma, the population of the energy levels for a particular species at a given position within the plasma is given by the Boltzmann equation [17]. The number of species $\mathrm{N}_{i}$ in the $i$ th excited state is given by

$$
N_{i}^{z}=N^{z} \frac{g_{i}^{z}}{U^{z}\left(T_{e}\right)} \exp \left(-\frac{E_{i}^{z}}{k_{B} T_{e}}\right) .
$$

Here, ionization state of the species is referred by the superscript $z$, whereas the neutral and singly ionized atomic species are represented by $z=0$ and $z=1$, respectively. $N_{i}^{z}, N^{z}, E_{i}^{z}$, and $g_{i}^{z}$ denote the population density at $i$ th level $\left(\mathrm{m}^{-3}\right)$, total population density or number density $\left(\mathrm{m}^{-3}\right)$, energy of upper energy level $(J)$, and degeneracy or statistical weight factor (dimensionless) of the upper level of the species with ionization $z$, respectively. $k_{B}$ is the Boltzmann's constant $\left(\mathrm{JK}^{-1}\right), T_{e}$ is the plasma temperature $(K)$, and $U^{z}\left(T_{e}\right)$ is the partition function of the species $\mathrm{z}$ at plasma temperature $T_{e}$. Assuming that the plasma is optically thin, the intensity of emitted radiation $\left(I_{i j}^{z}\right)$ of wavelength $\lambda_{i j}$ of the species $z$ in plasma can be written as

$$
I_{i j}^{z}=N_{i}^{z} A_{i j}^{z} \frac{h c}{4 \pi \lambda_{i j}},
$$

where $h$ is the Planck's constant $\left(\mathrm{Js}^{-1}\right), c$ is the speed of light $\left(\mathrm{ms}^{-1}\right)$ in vacuum, and $A_{i j}^{z}$ is the Einstein's coefficient of transition probability for spontaneous transition from upper level $i$ into lower lever $j$ of the species $z$. Substituting (2) in (3) gives

$$
I_{i j}^{z}=N^{z} A_{i j}^{z} \frac{h c}{4 \pi \lambda_{i j}} \frac{g_{i}^{z}}{U^{z}\left(T_{e}\right)} \exp \left(-\frac{E_{i}^{z}}{k_{B} T_{e}}\right) .
$$

Equation (4) can be transformed

$$
\ln \left(\frac{I_{i j}^{z} \lambda_{i j}}{A_{i j}^{z} g_{i}^{z}}\right)=\ln \left(\frac{h c N^{z}}{4 \pi U^{z}\left(T_{e}\right)}\right)-\frac{E_{i}^{z}}{k_{B} T_{e}} .
$$

By plotting the left side of (5) against the upper level energy $E_{i}^{z}$ (Boltzmann plot), a straight line is expected, whose slope yields the $T_{e}$. For high accuracy, the range of upper level energies of the so-called Boltzmann plot should be as large as possible [17]. The emission intensities of nine emission lines of vanadium from VOs LIP were used for constructing the Boltzmann plot. Table 2 lists the spectral lines used and their respective spectroscopic constants. The plasma temperatures are estimated within 10\% uncertainty mainly due to uncertainties in the transition probabilities for the lines and the measurement of the integrated line intensities used.

Figure 4 shows the temporal profile of $T_{e}$ for all the four VOs at a laser energy of $70 \mathrm{~mJ}$. The plasma temperature was found to decrease as the acquisition delay $\left(t_{d}\right)$ was increased. The plasma temperature as a function of $t_{d}$ is fitted with the power law [18-20]. At a laser energy of $70 \mathrm{~mJ}$, the fitted curves for VO $\left(T_{e} \sim t_{d}{ }^{-0.120 \pm 0.007}\right)$, $\mathrm{V}_{2} \mathrm{O}_{3}\left(T_{e} \sim t_{d}^{-0.120 \pm 0.007}\right), \mathrm{VO}_{2}\left(T_{e} \sim t_{d}{ }^{-0.127 \pm 0.008}\right)$, and $\mathrm{V}_{2} \mathrm{O}_{5}\left(T_{e} \sim t_{d}^{-0.129 \pm 0.004}\right)$ are shown in Figure 4. No drastic 


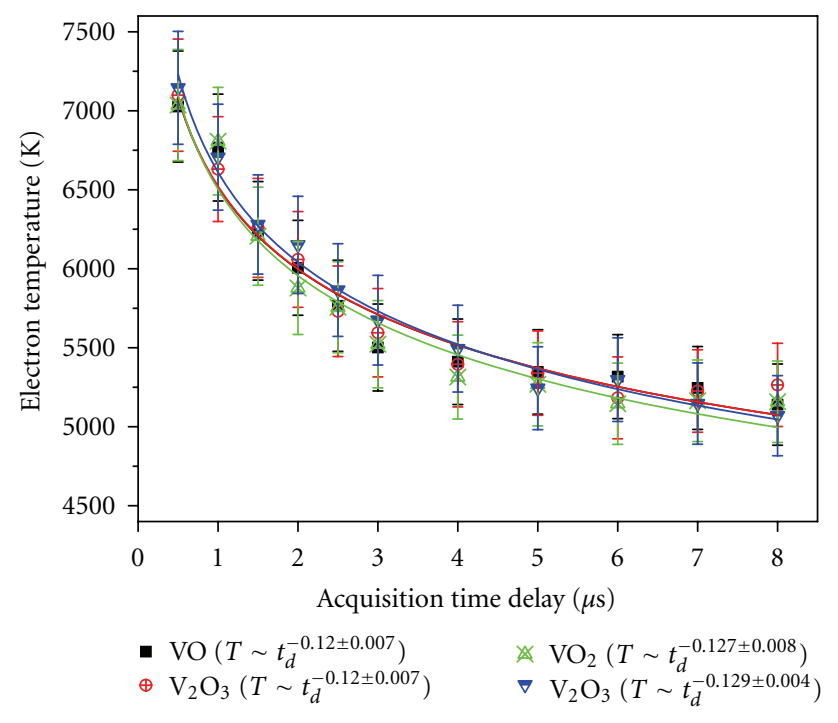

Figure 4: The plasma temperature $\left(T_{e}\right)$ as a function of acquisition time delay $(d)$ at $70 \mathrm{~mJ}$ for the different VOs.

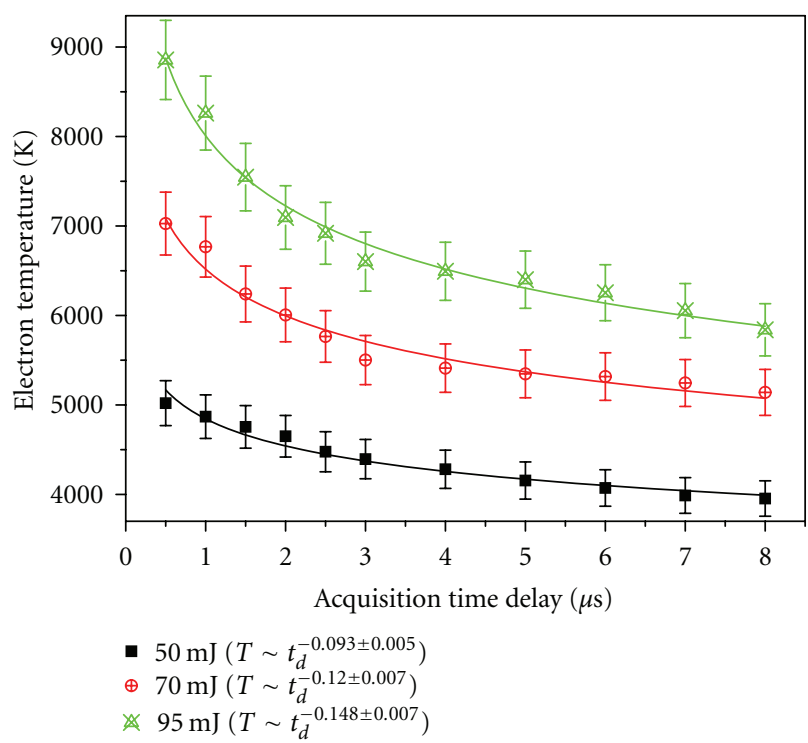

FIgure 5: The plasma temperature $\left(T_{e}\right)$ of VO as a function of acquisition time delay $\left(t_{d}\right)$ at laser energies of $50 \mathrm{~mJ}, 70 \mathrm{~mJ}$, and $95 \mathrm{~mJ}$.

plasma temperature difference was observed at any particular delay among the different VOs. The plasma temperature at the acquisition time delay of $500 \mathrm{~ns}$ was in the region of $7000 \mathrm{~K}$ which decreases to $5000 \mathrm{~K}$ at $8 \mu$ s. Similar temporal profile was observed for other laser energies of $50 \mathrm{~mJ}$ and $95 \mathrm{~mJ}$, except for differences in the magnitude of $T_{e}$. Figure 5 shows the temporal profile of plasma temperature for $\mathrm{VO}$ at different laser energies which was found to increase with laser energy. Based on Figures 4 and 5, the main features of plasma can be summarized as follows. (i) There is no significant difference in $T_{e}$ values obtained for different VOs at constant $t_{d}$ within the uncertainty of the calculation methodology.

(ii) For all the four VOs, the rate of decay in plasma temperature (i.e., $\mathrm{d} T_{e} / \mathrm{d} t_{d}$ ) is almost constant and hence can be assumed to be independent of matrix.

(iii) At a particular delay, with the increase of laser energy, $T_{e}$ increases. This is due to the fact that kinetic energy gained by the electrons increases as the deposited laser energy increases.

(iv) The temporal profile of $T_{e}$ also shows faster decay with increasing laser energy. This is expected, as higher $T_{e}$ means faster expansion of plasma leading to faster decrease of plasma temperature.

It may be noted that the space uniformity of the laser beam is not very crucial parameter in the present studies, since these plasma profiles (decay of $T_{e}$ and $N_{e}$ ) of different vanadium oxides have been recorded under the identical conditions.

3.3. Electron Density. The $N_{e}$ can be determined by determining Stark broadening of a well-isolated line in the plasma emission or by using combination of the Saha and Boltzmann equations. Under the present instrumental condition, due to poor resolving power, the application of Stark broadening method was not possible; hence, the latter method was used. Saha equation relates the densities of the subsequent ionization species for a plasma temperature $T_{e}$ [21]

$$
N_{e}=\frac{N^{z}}{N^{z+1}} \frac{2 U^{z+1}\left(T_{e}\right)}{U^{z}\left(T_{e}\right)}\left(\frac{2 \pi m k_{B} T_{e}}{h^{2}}\right)^{\frac{3}{2}} \exp \left(-\frac{E_{\infty}^{z}}{k_{B} T_{e}}\right) .
$$

Here, $N_{e}$ is the electron number density, $E_{\infty}^{z}$ is the ionization energy of species $z$ for an isolated system, $m$ is mass of electron $(\mathrm{kg})$.

From (4), the emitted intensity ratio of two emission lines from two species of two different states of ionization can be written as [21]

$$
\frac{I_{i j}^{z+1}}{I_{i j}^{z}}=\frac{N^{z+1}}{N^{z}} \frac{A_{i j}^{z+1} g_{i}^{z+1}}{A_{i j}^{z} g_{i}^{z}} \frac{\lambda_{i j}^{z} U^{z}\left(T_{e}\right)}{\lambda_{i j}^{z+1} U^{z+1}\left(T_{e}\right)} \exp \left(\frac{E_{i}^{z}-E_{i}^{z+1}}{k_{B} T_{e}}\right)
$$

Equation (7) can be rearranged as

$$
\frac{N^{z}}{N^{z+1}}=\frac{I_{i j}^{z}}{I_{i j}^{z+1}} \frac{A_{i j}^{z+1} g_{i}^{z+1}}{A_{i j}^{z} g_{i}^{z}} \frac{\lambda_{i j}^{z} U^{z}\left(T_{e}\right)}{\lambda_{i j}^{z+1} U^{z+1}\left(T_{e}\right)} \exp \left(\frac{E_{i}^{z}-E_{i}^{z+1}}{k_{B} T_{e}}\right) .
$$

Incorporating (8) in (6), the expression of $N_{e}$ can be written as:

$$
N_{e}=\frac{2 I_{i j}^{z}}{I_{i j}^{z+1}} \frac{A_{i j}^{z+1} g_{i}^{z+1}}{A_{i j}^{z} g_{i}^{z}} \frac{\lambda_{i j}^{z}}{\lambda_{i j}^{z+1}}\left(\frac{2 \pi m k_{B} T_{e}}{h^{2}}\right)^{3 / 2} \exp \left(\frac{E_{i}^{z+}-E_{i}^{z+1}-E_{\infty}^{z}}{k_{B} T_{e}}\right) .
$$

All the right-hand side parameters in (9) can either be measured or obtained from compilation of atomic properties. The electron densities were estimated within $10 \%$ uncertainty similar to $T_{e}$. 


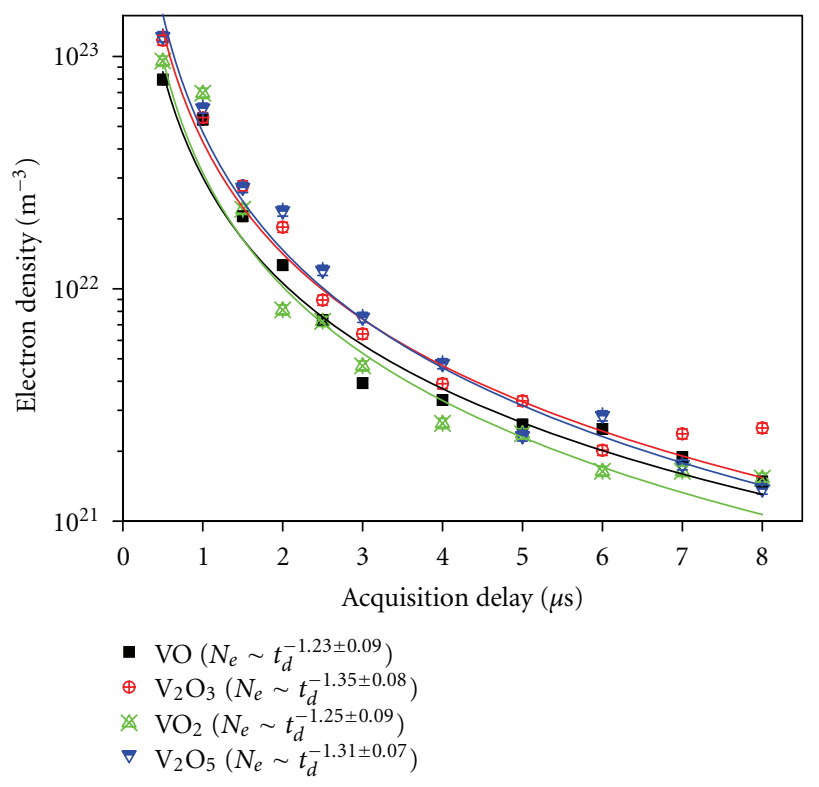

FIGURE 6: The electron density $\left(N_{e}\right)$ as a function of acquisition time delay $\left(t_{d}\right)$ at $70 \mathrm{~mJ}$ for the different VOs.

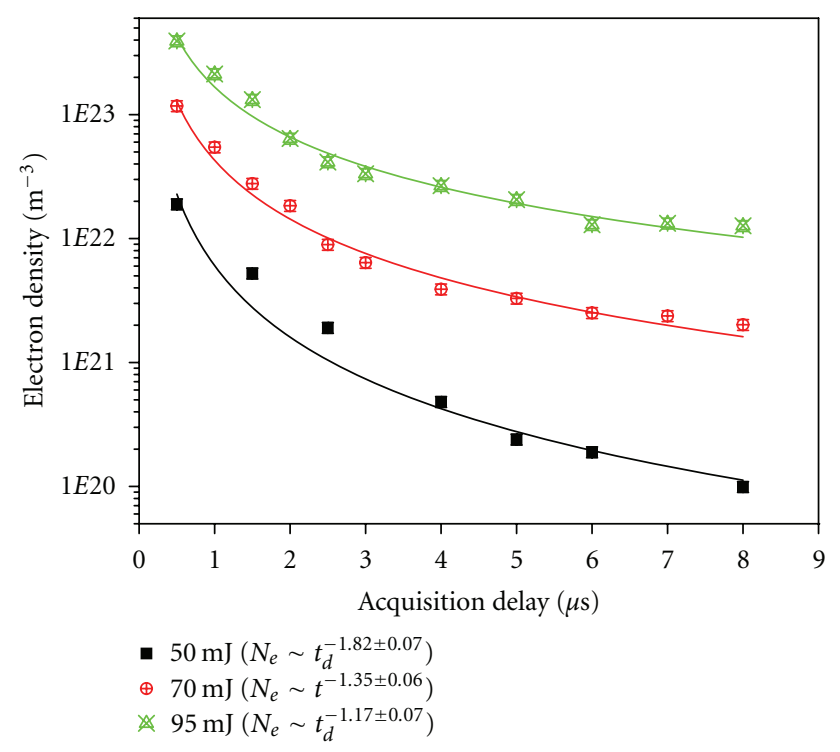

FIgure 7: The electron density $\left(N_{e}\right)$ of $\mathrm{V}_{2} \mathrm{O}_{3}$ as a function of acquisition time delay at laser energies of $50 \mathrm{~mJ}, 70 \mathrm{~mJ}$, and $95 \mathrm{~mJ}$.

The $N_{e}$ as a function of $t_{d}$ was found to have the same trend of temporal profile in all the VOs, that is, $N_{e}$ decreasing with the increase of $t_{d}$ following a power law. Figure 6 shows a typical temporal profile of $N_{e}$ with $t_{d}$ at $70 \mathrm{~mJ}$ for all the VOs. $N_{e}$ decreases fast in the early stage of plasma formation, but after $\sim 3 \mu \mathrm{s}$, there is no appreciable decrease. $N_{e}$ for $\mathrm{V}_{2} \mathrm{O}_{3}$ decreased from $\sim 1 \times 10^{23} \mathrm{~m}^{-3}$ to $\sim 6 \times 10^{21} \mathrm{~m}^{-3}$ during of 0.5 to $3 \mu \mathrm{s}$, and this value further reduced to $\sim 1 \times 10^{21} \mathrm{~m}^{-3}$ at $8 \mu \mathrm{s}$. There is no noticeable difference in the $N_{e}$ among the samples. Similar temporal profiles were also obtained at $50 \mathrm{~mJ}$ and $95 \mathrm{~mJ}$ laser energies except for different magnitudes of $N_{e} . N_{e}$ as a function of $t_{d}$ was fitted with the power law similar to that for $T_{e}$. The power law fittings for a laser energy of $70 \mathrm{~mJ}$ for $\mathrm{VO}\left(N_{e} \sim t_{d}{ }^{-1.28 \pm 0.09}\right)$, $\mathrm{V}_{2} \mathrm{O}_{3}\left(N_{e} \sim t_{d}^{-1.35 \pm 0.07}\right), \mathrm{VO}_{2}\left(N_{e} \sim t_{d}^{-1.25 \pm 0.09}\right)$, and $\mathrm{V}_{2} \mathrm{O}_{5}\left(N_{e} \sim t_{d}{ }^{-1.31 \pm 0.07}\right)$ are also shown in Figure 6. It can be seen that similar to $T_{e}$, at a particular $t_{d}$, the rate of decay of $N_{e}$ is independent of type of oxide similar to the $T_{e}$ temporal followup. The dependency of rate of decay with laser energy was examined for $\mathrm{V}_{2} \mathrm{O}_{3}$ and was found to be faster with reduction in laser energy incident (Figure 7). This is the reverse of that observed for $T_{e}$. With increase of laser energy, the amount of material ablated increases. The $N_{e}$ can be sustained for a longer time; hence, the rate of decay of $N_{e}$ decreases with increasing ablation. It is interesting to note that the rate of decay of $N_{e}$ is much faster than that of $T_{e}$ for all the vanadium oxides. This clearly emphasizes the fact that initially, the $N_{e}$ is very high, and radiative emission will be a dominating emitting source. Therefore, to obtain good elemental signal, suitable acquisition delay must be employed in LIBS.

\section{Conclusion}

Using fundamental Nd:YAG laser, LIPs of four different vanadium oxides were investigated. The temporal profiles of ionic or atomic lines have different patterns for different oxides but main LIP parameters, that is, $T_{e}$ and $N_{e}$, do not show any appreciable differences in their temporal profiles. $T_{e}$ and $N_{e}$ were measured at different acquisition time delays for all the four VOs. It was found that in all VOs, the rate of decay of both $T_{e}$ and $N_{e}$ follows a power law and is constant at a particular plasma temperature. The rate of decay of $N_{e}$ is much faster than that of $T_{e}$ in the initial period, that is, $0-3 \mu$ s region. With the increase of laser energy, rate of decay of $T_{e}$ increases for all the VOs, but for $N_{e}$, the reverse trend is observed. The studies reported in this work are useful to optimize different parameters, for example, laser energy, acquisition delay, and so forth, for LIBS analysis of highpurity vanadium-based materials.

\section{Acknowledgment}

The authors are thankful to Dr. V. Venugopal, Director, Radiochemistry and Isotope Group, B.A.R.C., for his constant support and encouragement in LIBS work.

\section{References}

[1] G. M. Weyl, "Physics of laser-induced breakdown: an update," in Laser-Induced Plasmas and Applications, L. J. Radziemski and D. A. Cremers, Eds., Marcel Dekker, New York, NY, USA, 1989.

[2] C. Geertsen, A. Briand, F. Chartier et al., "Comparison between infrared and ultraviolet laser ablation at atmospheric pressure-implications for solid sampling inductively coupled plasma spectrometry," Journal of Analytical Atomic Spectrometry, vol. 9, no. 1, pp. 17-22, 1994.

[3] M. A. Shannon, X. L. Mao, A. Fernandez, W. T. Chan, and R. E. Russo, "Laser ablation mass removal versus incident power 
density during solid sampling for inductively coupled plasma atomic emission spectroscopy," Analytical Chemistry, vol. 67, no. 24, pp. 4522-4529, 1995.

[4] M. Autin, A. Briand, P. Mauchien, and J. M. Mermet, "Characterization by emission spectrometry of a laser-produced plasma from a copper target in air at atmospheric pressure," Spectrochimica Acta-Part B, vol. 48, no. 6-7, pp. 851-862, 1993.

[5] B. C. Castle, K. Visser, B. W. Smith, and J. D. Winefordner, "Spatial and temporal dependence of lead emission in laserinduced breakdown spectroscopy," Applied Spectroscopy, vol. 51, no. 7, pp. 1017-1024, 1997.

[6] L. M. Cabalin and J. J. Laserna, "Experimental determination of laser induced breakdown thresholds of metals under nanosecond Q-switched laser operation," Spectrochimica Acta-Part B, vol. 53, no. 5, pp. 723-730, 1998.

[7] S. Nasrazadani and H. Namduri, "Study of phase transformation in iron oxides using laser induced breakdown spectroscopy," Spectrochimica Acta-Part B, vol. 61, no. 5, pp. 565-571, 2006.

[8] R. S. Adrain and J. Watson, "Laser microspectral analysis: a review of principles and applications," Journal of Physics D, vol. 17, no. 10, article 004, pp. 1915-1940, 1984.

[9] H. R. Griem, Principles of Plasma Spectroscopy, Cambridge University Press, Cambridge, UK, 1997.

[10] D. H. Lowndes, D. B. Geohegan, A. A. Puretzky, D. P. Norton, and C. M. Rouleau, "Synthesis of novel thin-film materials by pulsed laser deposition," Science, vol. 273, no. 5277, pp. 898903, 1996.

[11] M. A. Heald and C. B. Wharton, Plasma Diagnostics with Microwaves, Wiley, NewYork, NY, USA, 1965.

[12] T. Mochizuki, K. Hirata, H. Ninomiya et al., "Time-resolved electron density measurements in an ArF excimer laser discharge," Optics Communications, vol. 72, no. 5, pp. 302305, 1989.

[13] S. M. Cameron, M. D. Tracy, and J. F. Camacho, "Electron density and temperature contour plots from a laser-produced plasma using collective ultraviolet Thomson scattering," IEEE Transactions on Plasma Science, vol. 24, no. 1, pp. 45-46, 1996.

[14] R. W. P. McWhirter, "Spectral intensities," in Plasma Diagnostic Techniques, R. H. Huddlestone and S. L. Leonard, Eds., Academic Press, 1965.

[15] http://www.nist.gov/pml/data/asd.cfm.

[16] I. V. Kityk, "Photoinduced non-linear optical diagnostic of $\mathrm{SiN}_{\mathrm{x}} \mathrm{O}_{\mathrm{y}} / \mathrm{Si}^{<} 111^{>}$interfaces," Optics and Lasers in Engineering, vol. 35, no. 4, pp. 239-250, 2001.

[17] W. Lochte-Holtgreven, Ed., Plasma Diagnostics, NorthHolland, 1968.

[18] N. M. Shaikh, B. Rashid, S. Hafeez, Y. Jamil, and M. A. Baig, "Measurement of electron density and temperature of a laserinduced zinc plasma," Journal of Physics D, vol. 39, no. 7, pp. 1384-1391, 2006.

[19] S. S. Harilal, C. V. Bindhu, R. C. Issac, V. P. N. Nampoori, and C. P. G. Vallabhan, "Electron density and temperature measurements in a laser produced carbon plasma," Journal of Applied Physics, vol. 82, no. 5, pp. 2140-2146, 1997.

[20] A. Bogaerts and Z. Chen, "Effect of laser parameters on laser ablation and laser-induced plasma formation: a numerical modeling investigation," Spectrochimica Acta-Part B, vol. 60, no. 9-10, pp. 1280-1307, 2005.

[21] H. R. Griem, Plasma Spectroscopy, McGraw Hill, New York, NY, USA, 1964. 

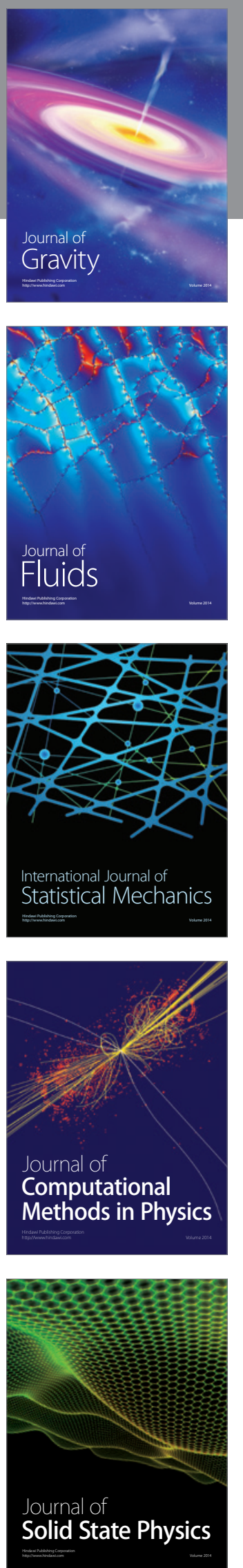

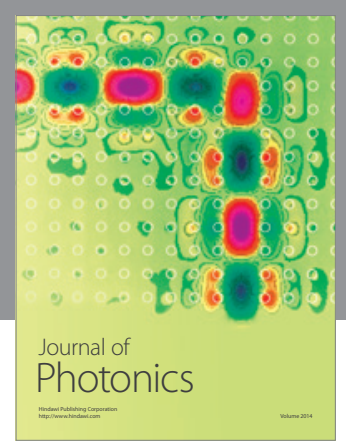

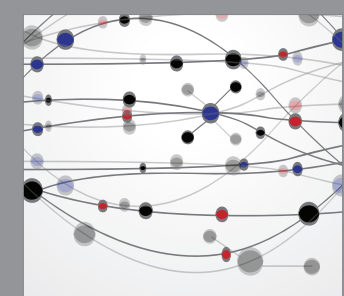

The Scientific World Journal
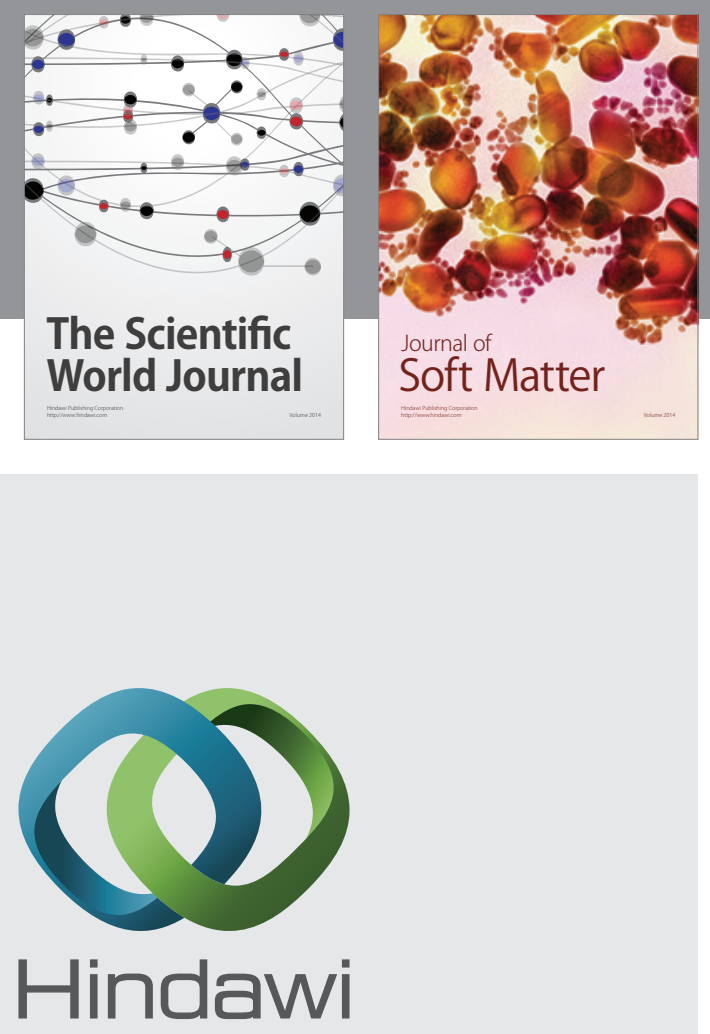

Submit your manuscripts at

http://www.hindawi.com
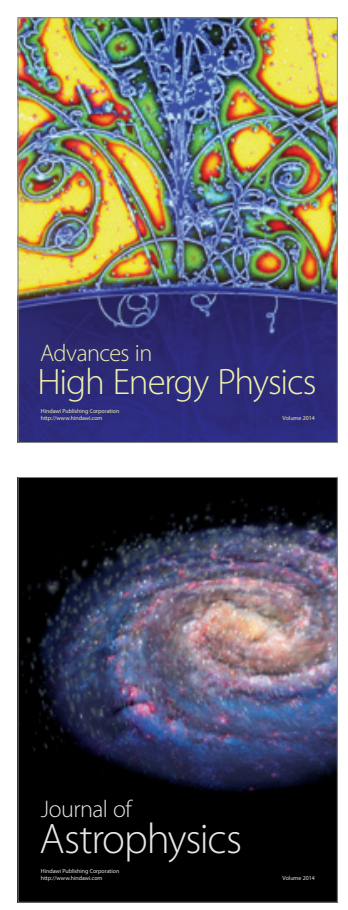
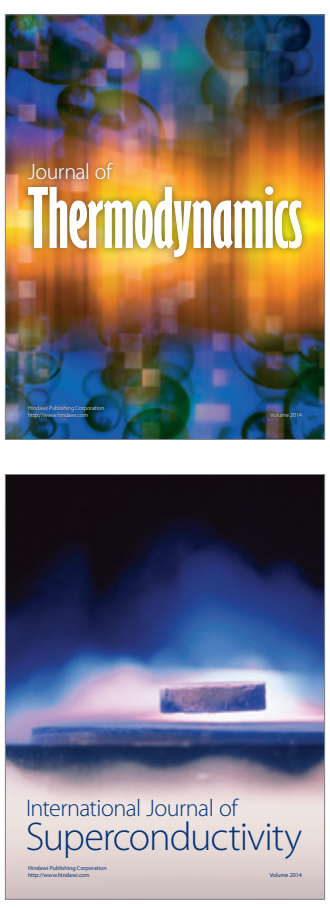
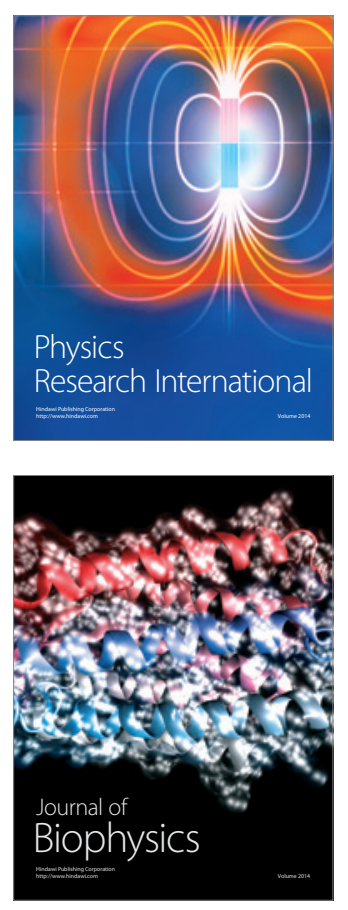
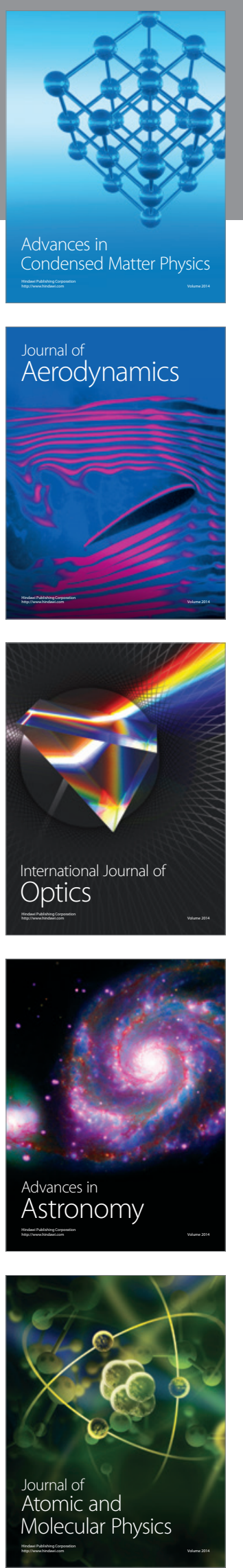